\title{
Ion pump ATPase inhibitors block the fertilization of zona-free mouse oocytes by acrosome-reacted spermatozoa*
}

\author{
B. E. Talansky†, P. E. Barg $\ddagger$ and J. W. Gordon $\ddagger \S$ \\ Department of $\ddagger$ Obstetrics, Gynecology and Reproductive Science, $\nmid$ Physiology and Biophysics and \\ $\S$ Biochemistry, Mt Sinai School of Medicine, 1 Gustave L. Levy Place, New York, NY 10029, \\ U.S.A.
}

\begin{abstract}
Summary. The acrosome reaction was induced in nearly $100 \%$ of mouse spermatozoa with dibutyryl cyclic guanosine monophosphate (dbcGMP) before ouabain treatment. Acrosome-reacted spermatozoa could not penetrate the zona pellucida, but readily fertilized zona-free eggs. Exposure to ouabain at concentrations as low as $10^{-7} \mathrm{M}$ had a noticeable inhibitory effect upon fertilization. Similar results were obtained with a second ATPase inhibitor, digoxin. These results show that ion-pump inhibitors block the union of gametes which are otherwise fully competent to fertilize. These findings suggest that a membrane potential maintained by ion pumps is a necessary prerequisite for gamete fusion.
\end{abstract}

\section{Introduction}

After its release from the testis, the ejaculated mammalian spermatozoon must undergo additional changes before it is able to unite with the oocyte. Spermatozoa must become capacitated (Austin, 1951, 1952; Chang, 1951) and complete the acrosome reaction (Austin \& Bishop, 1958) before fertilizing the egg. These prerequisites have been elucidated by studies of populations of ejaculated spermatozoa. However, it is more difficult to define the precise characteristics of the single spermatozoon which reaches the egg membrane and fuses with it. This is because only one of millions of spermatozoa in the ejaculate actually fertilizes the oocyte, and because the ejaculate is a heterogeneous population of cells, many of which may never become competent to fertilize.

To assess the fertilizability of individual spermatozoa in the mouse ejaculate we have used procedures which yield a population of uniformly acrosome-reacted spermatozoa, and have examined the ability of individual cells selected randomly from these preparations to fertilize the oocyte after mechanical insertion under the zona pellucida (Barg et al., 1985). Capacitated, acrosome-reacted spermatozoa, if rendered immotile, were unable to fertilize the egg when inserted under the zona pellucida. A striking observation in these experiments was that mechanical manipulation of the distal flagellar membrane resulted in an immediate cessation of sperm motility, suggesting that mechanical contact with the flagellum induced a change in membrane properties which then rendered the spermatozoa nonfunctional.

To test this possibility, we exposed acrosome-reacted spermatozoa to membrane ion-pump inhibitors that would not interfere with motility but which would alter the sperm membrane potential. Previous experiments had shown that ouabain, a potent inhibitor of $\mathrm{Na}^{+}-\mathrm{K}^{+}$ATPases, interfered with sperm motility only at relatively high concentrations, and stimulated motility at low concentrations in some organisms (Nelson \& McGrady, 1981). Because ouabain had also been

\footnotetext{
*Reprint requests to Dr J. W. Gordon.
} 
shown to block the acrosome reaction in hamster spermatozoa (Mrsny \& Meizel, 1981), it was necessary to obtain a population of spermatozoa that was extensively acrosome-reacted before ouabain exposure. We accordingly used dibutyryl cyclic guanosine monophosphate (dbcGMP) to produce uniformly acrosome-reacted, motile spermatozoa, and examined the effect of ouabain and digoxin, another $\mathrm{Na}^{+}-\mathrm{K}^{+}$ATPase inhibitor, on the ability of such spermatozoa to fertilize the zona-free mouse oocyte.

\section{Materials and Methods}

Mice used: egg and sperm recovery. CD-1 female mice were obtained from Charles River Breeding Laboratories. B6D2 $\mathrm{f}_{1}$ male mice were purchased from the Jackson Laboratory, Bar Harbor, Maine. Mice were maintained on a $14 \mathrm{~h}$ light: $10 \mathrm{~h}$ dark schedule. CD-1 females were induced to superovulate at 6 weeks of age with 5 i.u. PMSG (Gestyl: Organon, W. Orange, NJ) at 16:00 h followed $48 \mathrm{~h}$ later by 2.5 i.u. hCG (Pregnyl: Organon). Females were killed at $08: 00 \mathrm{~h}$ the morning after injection and the oviducts were removed into bicarbonate-buffered medium (Hoppe \& Pitts, 1973) supplemented with $4 \mathrm{mg}$ bovine serum albumin/ml. Oviducts were opened with forceps and unfertilized eggs were expressed into depression slides containing the same medium supplemented with $1 \mathrm{mg}$ hyaluronidase/ml. After the granulosa cells were dispersed, the eggs were removed and washed in $2 \mathrm{ml}$ culture medium equilibrated with $5 \%$ $\mathrm{CO}_{2}$ in air at $37^{\circ} \mathrm{C}$. The standard medium used was a modification (Thadani, 1982) of that of Hoppe \& Pitts (1973) in which sodium chloride was raised to $5.97 \mathrm{~g} / \mathrm{l}$. The final measured osmolarity was $255 \mathrm{mosmol} / \mathrm{kg}$.

After rinsing in culture medium the oocytes were collected and treated briefly with acid Tyrode's solution $\mathrm{pH} 2 \cdot 3$ to remove the zonae pellucidae. The eggs were then thoroughly rinsed and loaded into $20 \mu \mathrm{l}$ microdrops under mineral oil. Medium in the microdrops was supplemented with various concentrations of ouabain; control oocytes were loaded into medium without ouabain. Spermatozoa were added to microdrops to give a final concentration of $1 \times 10^{6}$ motile spermatozoa/ml. Fertilization was assessed by several criteria. In most cases fertilization was obvious by the presence of at least 3 pronuclei resulting from polyspermic fertilization of the zona-free eggs. Fertilization was also manifest by the release of the second polar body and the appearance of female and male pronuclei. Spontaneous oocyte activation was distinguished from true fertilization by the observation of second polar body release, and by the fact that the male strain used in these experiments produces pronuclei with several nucleoli, while the female pronucleus has a single large nucleolus.

Preparation of spermatozoa. Spermatozoa were expressed from each distal vas deferens and cauda epididymidis of male mice and placed in medium supplemented with $60 \%$ sodium lactate syrup $(4 \mu 1 / \mathrm{ml})$. After prior incubation to allow dispersal and capacitation, spermatozoa were divided into experimental groups for further treatment as described below.

Induction of the acrosome reaction in vitro. It has been shown by electron microscopy that incubation for $2 \mathrm{~h}$ is by itself sufficient for induction of the acrosome reaction in 20-30\% of mouse spermatoza (Barg et al., 1985). However, when such sperm preparations are used to inseminate eggs, the possibility cannot be excluded that other spermatozoa which undergo the acrosome reaction after insemination are those which actually fertilize the oocyte. Since ouabain, the ion-pump inhibitor used in this study, is known to block the acrosome reaction (Mrsny \& Meizel, 1981; Mrsny et al. 1984 ) it was necessary to establish that ouabain did not prevent fertilization by blocking the acrosome reaction in spermatozoa that were not acrosome reacted at the end of the incubation period. Accordingly, a series of experiments was performed wherein the spermatozoa were exposed to dbcGMP before ouabain treatment. The concentrations and exposure times applied to guinea-pig spermatozoa (Santos-Sacchi \& Gordon, 1980) were used in the present experiments with the modification that mouse spermatozoa were preincubated to achieve optimal capacitation before induction of the acrosome reaction by dbcGMP.

After incubation for $1 \mathrm{~h} 30 \mathrm{~min}$ or $2 \mathrm{~h}$ in capacitation medium, spermatozoa were transferred to medium supplemented with $12 \mathrm{mM}$-dbcGMP and $10 \mathrm{~mm}$-imidazole, and incubated for an additional $30 \mathrm{~min}$. This protocol was found to induce maximal acrosome reactions as demonstrated by electron microscopy.

Tests of zona penetration by dbcGMP-treated spermatozoa. After exposure to dbcGMP, spermatozoa were tested for their ability to penetrate the zona pellucida, by performing inseminations of zona-intact oocytes retrieved after superovulation. As a test of the in-vitro fertilization procedures, pairwise inseminations with untreated spermatozoa were conducted. Differences in fertilization rates after insemination with equal numbers of treated and control spermatozoa were then compared.

Treatment with ion-pump inhibitors. After incubation in culture medium or culture medium followed by dbcGMP treatment, spermatozoa were centrifuged at $600 \mathrm{~g}$ for $10 \mathrm{~min}$. They were then resuspended in culture medium supplemented with 10 times greater concentrations of ouabain such that the final concentration was equal to that desired for insemination. Spermatozoa were incubated in ouabain for $20 \mathrm{~min}$ before insemination. In a single experiment, spermatozoa were treated with $100 \mu \mathrm{g}$ digoxin/ml for the same $20 \mathrm{~min}$ period and then combined with oocytes at this same concentration of digoxin.

In experiments in which spermatozoa were not exposed to dbcGMP, they were incubated for $2 \mathrm{~h}$ or $2 \mathrm{~h} 30 \mathrm{~min}$ in culture medium and $20 \mathrm{~min}$ in ouabain. Control groups exposed neither to dbcGMP nor ouabain were incubated for 
$2 \mathrm{~h} 20 \mathrm{~min}$ or $2 \mathrm{~h} 50 \mathrm{~min}$ in normal medium. Thus all sperm preparations were preincubated for the same period before insemination.

Assessment of sperm count and motility. Spermatozoa were counted and their motility assessed by diluting incubated preparations and placing them on a haemocytometer. Spermatozoa were examined by visual inspection for their degree of active motility and for forward progression across the haemocytometer grid.

Preparation of spermatozoa for electron microscopy. After capacitation, control and dbcGMP-treated spermatozoa were centrifuged for $10 \mathrm{~min}$ at $600 \mathrm{~g}$ and the pellet was resuspended in $3 \mathrm{ml}$ sodium cacodylate buffer. After centrifugation for an additional $10 \mathrm{~min}$, the supernatant was removed and the pellets were resuspended in a $1-1.5 \mathrm{ml}$

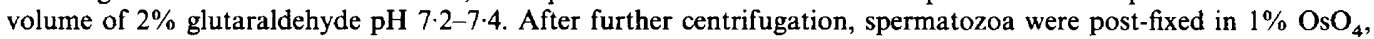
dehydrated in ethanol and embedded in Spurr's resin. Thin sections were examined in a Philips 300 electron microscope at a voltage of $60 \mathrm{kV}$. The percentage of acrosome-reacted spermatozoa was determined by direct examination and scoring of at least 100 cells under low magnification.

Tests for ouabain toxicity. This test was conducted by first determining the minimal concentration of ouabain necessary for complete inhibition of fertilization $\left(10^{-5} \mathrm{M}\right)$. Spermatozoa and eggs were then preincubated with 100 times this minimal amount $\left(10^{-3} \mathrm{M}\right)$. The spermatozoa were washed twice by gentle centrifugation and resuspension in culture medium. Eggs were washed by serial transfer into several dishes of normal medium. Inseminations were then carried out in normal medium as described above.

Statistical testing. Fertilization rates were calculated as the number of oocytes fertilized divided by the number inseminated. Statistical significance of observed differences in fertilization rates was determined by $\chi^{2}$ analysis using 1 degree of freedom. Differences were considered statistically significant at a $P$ value of less than 0.05 .

\section{Results}

\section{Effect of dbcGMP treatment}

Exposure of mouse spermatozoa to dbcGMP effectively induced the acrosome reaction. Examination of spermatozoa by electron microscopy showed that at least $90 \%$ (95 of 100 spermatozoa examined) were acrosome reacted. Figure 1 shows a typical spermatozoon after exposure to this compound. The acrosome reaction is completed and the normal cellular architecture is preserved. In control preparations incubated without dbcGMP, only $20-30 \%$ of the spermatozoa were acrosome reacted.

Dibutyryl cGMP did not impair either the motility of spermatozoa or their ability to fertilize zona-free oocytes. The rate of fertilization after dbcGMP treatment was not significantly different from that of control untreated spermatozoa (Table 1).

\section{Penetration of zona pellucida by acrosome-reacted spermatozoa}

Because spermatozoa were induced to undergo the acrosome reaction before insemination, their ability to fertilize zona-intact oocytes provided a rigorous test of the importance of an intact acrosome at the time of zona contact to the fertilization process. Treatment with dbcGMP drastically reduced the fertilization rate of zona-intact mouse oocytes $(4 / 286,1 \cdot 4 \%)$ relative to the rate obtained with untreated spermatozoa $(399 / 1595,25 \%)$. This result reinforces the electron microscopic finding that dbcGMP-treated spermatozoa are nearly $100 \%$ acrosome reacted, and confirms previous suggestions (Saling et al., 1979; Florman \& Storey, 1982) that otherwise normal mouse spermatozoa that have undergone the acrosome reaction before zona contact cannot fertilize oocytes.

\section{Spermatozoa capacitated for $2 \mathrm{~h}$ before ouabain treatment}

After $2 \mathrm{~h}$ of capacitation followed by $20 \mathrm{~min}$ of ouabain treatment, fertilization was inhibited in ouabain concentrations as low as $10^{-6} \mathrm{M}$. Of 282 oocytes 92 were fertilized (33\%). This rate was significantly lower $(P<0.001)$ than in control inseminations without ouabain, in which the fertilization rate was $100 \%$. Spermatozoa treated with dbcGMP proved more resistant to ouabain 




Fig. 1. Electron micrograph of a typical spermatozoon from dbcGMP-treated preparations. The acrosome reaction is complete. $\times 36000$.

inhibition than spermatozoa not treated (Table 1). Thus in these experiments, dbcGMP reduced the sensitivity of spermatozoa to ouabain.

\section{Spermatozoa capacitated for $2 \mathrm{~h} 30 \mathrm{~min}$ before ouabain treatment}

These spermatozoa were more sensitive to ouabain than those capacitated for only $2 \mathrm{~h}$. Fertilization was significantly inhibited at $10^{-6} \mathrm{M}$, with only $35 \%$ of eggs fertilized (Table 1). At $10^{-7} \mathrm{M}$ the fertilization rate was $65 \%$, still significantly lower than that of untreated spermatozoa $(65 \% \mathrm{vs}$ 
Table 1. Mouse oocytes inseminated after capacitation of spermatozoa for $2 \mathrm{~h}$ or $2 \mathrm{~h} 30 \mathrm{~min}$

\begin{tabular}{cccccc}
\hline $\begin{array}{c}\text { Capacitation } \\
\text { time }\end{array}$ & $\begin{array}{c}\text { Ouabain conc. } \\
(\mathrm{mol} / 1)\end{array}$ & Ouabain & dbcGMP & $\begin{array}{c}\text { No. of oocytes } \\
\text { fertilized/no. } \\
\text { inseminated }\end{array}$ & $(\%)$ \\
\hline $2 \mathrm{~h}$ & $10^{-3}$ & + & - & $0 / 8$ & $(0)^{*}$ \\
& $10^{-4}$ & + & - & $0 / 22$ & $(0)^{* *}$ \\
& $10^{-5}$ & + & - & $1 / 22$ & $(5)^{* *}$ \\
& $10^{-5}$ & + & + & $0 / 29$ & $(0)^{* *}$ \\
& $10^{-6}$ & + & - & $92 / 282$ & $(33)^{* *}$ \\
$2 \mathrm{~h} 30 \mathrm{~min}$ & $10^{-6}$ & + & + & $208 / 270$ & $(77)$ \\
& 0 & - & - & $34 / 34$ & $(100)$ \\
& 0 & - & + & $109 / 125$ & $(87)$ \\
\hline $10^{-5}$ & + & - & $0 / 41$ & $(0)^{* *}$ \\
& $10^{-5}$ & + & + & $0 / 18$ & $(0)^{* *}$ \\
& $10^{-6}$ & + & - & $37 / 105$ & $(35)^{* *}$ \\
& $10^{-6}$ & + & + & $37 / 95$ & $(39)^{* *}$ \\
& $10^{-7}$ & + & - & $46 / 71$ & $(65)^{* *}$ \\
& $10^{-7}$ & + & - & $29 / 52$ & $(56)^{* *}$ \\
& 0 & - & + & $23 / 24$ & $(96)$ \\
& 0 & - & & $19 / 19$ & $(100)$ \\
\hline
\end{tabular}

${ }^{*} P<0.05 ;{ }^{* *} P<0.001$ compared with control values $\left(\chi^{2}\right.$ tests).

$96 \%, P<0.01$ ). Spermatozoa treated with dbcGMP were also inhibited at $10^{-7} \mathrm{M}$-ouabain, with $56 \%$ of the eggs fertilized. In contrast to spermatozoa capacitated for $2 \mathrm{~h}$, those capacitated for $2 \mathrm{~h}$ $30 \mathrm{~min}$ were not more resistant to ouabain than controls. At $10^{-6}$ or $10^{-7} \mathrm{M}$ the fertilization rate was not higher after treatment with dbcGMP than without exposure to this compound.

\section{Ouabain blocks fertilization, but not sperm attachment}

In control inseminations spermatozoa bound rapidly to zona-free oocytes. After oocyte activation the number of bound spermatozoa was reduced. Activated oocytes could therefore be readily identified before the appearance of a second polar body or pronuclei, and a relative paucity of bound spermatozoa was evident at the time eggs were scored for fertilization (Fig. 2a). As with the controls, inseminations in the presence of ouabain were characterized by a rapid binding of numerous spermatozoa to the egg surface. However, oocyte activation never took place. Neither second polar bodies nor pronuclei appeared. At the end of the fertilization period, large numbers of spermatozoa were still seen attached to the oocyte surface (Fig. 2b).

\section{Ouabain does not block fertilization by non-specific toxic effects upon the gametes}

As a test for ouabain toxicity, experiments were conducted wherein spermatozoa or eggs were preincubated in ouabain adjusted to concentrations at least 100 times greater $\left(10^{-3} \mathrm{M}\right)$ than those needed to inhibit fertilization totally, and 10000 times greater than concentrations needed to impair fertilization noticeably. They were then washed and inseminated in normal medium. After treatment of spermatozoa in this manner, 133/137 (97\%) of zona-free eggs were fertilized, while treatment of eggs followed by washing resulted in $43 / 43(100 \%)$ fertilization rate. These high concentrations therefore had no inhibitory effect upon spermatozoa or eggs. 

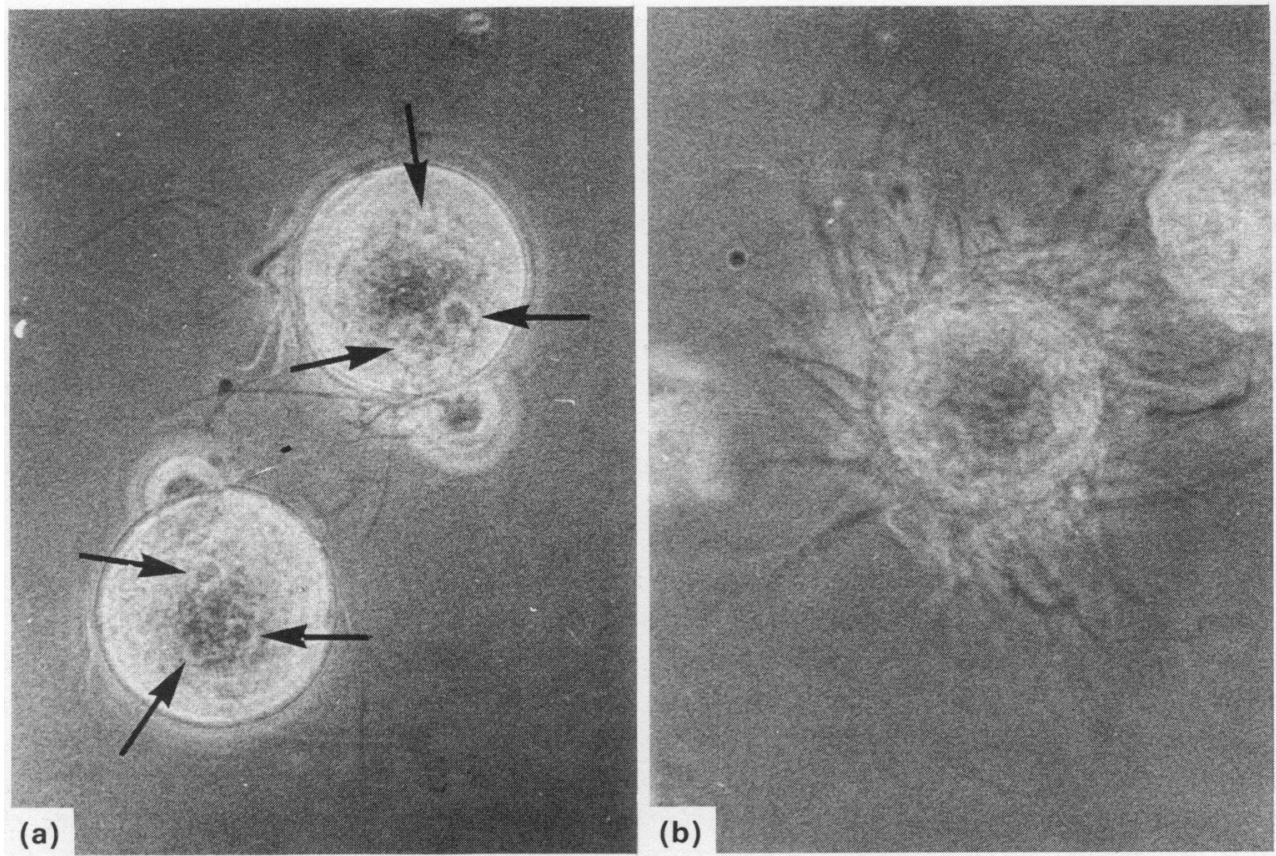

Fig. 2. Zona-free oocytes inseminated with (a) or without (b) ouabain. At this concentration $\left(10^{-5} \mathrm{M}\right)$ fertilization is completely blocked, but extensive binding of the spermatozoon to the vitellus still takes place (b). Signs of fertilization present in all control oocytes shown include release of the second polar body, a block to sperm binding and formation of pronuclei. Two spermatozoa have fused with each oocyte in the control group, with resultant formation of 3 pronuclei (arrows).

It was not possible to rule out effects of ouabain on oocytes as well as spermatozoa. That ouabain has an effect on spermatozoa was demonstrated by the observation that spermatozoa preincubated for $2 \mathrm{~h}$ were more resistant to $10^{-6} \mathrm{M}$ ouabain than were those capacitated for $2 \mathrm{~h}$ $30 \mathrm{~min}$. We were unable to demonstrate a direct effect on the oocyte because oocytes were fertilized normally after washing and insemination regardless of the protocol of ouabain exposure.

\section{Discussion}

We have used dbcGMP to induce the acrosome reaction in nearly $100 \%$ of mouse spermatozoa and have demonstrated that such spermatozoa are normal in their ability to fertilize oocytes. This acrosome-reaction induction protocol thus contrasts with calcium ionophore treatment (which renders spermatozoa immotile) and allowed separation of the inhibitory effects of ouabain on acrosome-reaction induction from its potential action upon gamete fusion itself. The results presented show that, when exposed to very low concentrations of ouabain, acrosome-reacted spermatozoa are unable to fertilize zona-free mouse oocytes. Because in other systems inhibitors such as ouabain lead to alterations in membrane potential, these findings suggest that the gamete membranes require a potential in order for fusion to take place.

It was important in these experiments to establish that the spermatozoa were acrosome reacted before ouabain treatment. Previous work with the hamster has demonstrated that ouabain will block the acrosome reaction and thereby indirectly block fertilization (Mrsny \& Meizel, 1981). It is 
well established that incubation of mouse spermatozoa in appropriate media for $1-2 \mathrm{~h}$ induces the acrosome reaction in sufficient numbers of spermatozoa for fertilization to take place (Rogers, 1978; Thadani, 1982). The present experiments agree with these data, showing that spermatozoa incubated for $2 \mathrm{~h} 20 \mathrm{~min}$ and then exposed to zona-free oocytes readily fertilize the egg (Table 1). However, examination of mouse spermatozoa by electron microscopy (Barg et al., 1985) has demonstrated that, in these preparations, only $20-30 \%$ of the spermatozoa are acrosome reacted. When such preparations are used for in-vitro fertilization, it is not possible to determine whether the $20-30 \%$ of spermatozoa that are acrosome reacted at the time of insemination are the same spermatozoa which fuse with the oocyte. Many additional spermatozoa could undergo the acrosome reaction after insemination and fertilize the ovum. This problem has been encountered previously in experiments examining the effect of trypsin inhibitors on fertilization of zona-free mouse oocytes. Although these compounds blocked fertilization, it was not possible to rule out that such blockage resulted from interference with the acrosome reaction (Wolf, 1977).

The present study has solved this ambiguity by treating spermatozoa with dbcGMP before insemination. Electron microscopic examination has shown that at least $90 \%$ of the spermatozoa are acrosome-reacted after dbcGMP treatment. Further, the acrosome reaction induced by dbcGMP is physiologically normal. Zona-free inseminations clearly demonstrate that spermatozoa treated with dbcGMP fertilize oocytes as efficiently as do spermatozoa treated by incubation alone. Because only $5-10 \%$ of spermatozoa retain their acrosomes after dbcGMP exposure, the results argue strongly that ouabain did not block fertilization by interfering with the acrosome reaction.

It was also necessary to demonstrate that ouabain did not block fertilization via non-specific toxic effects. This possibility was tested by preincubating eggs and spermatozoa in concentrations of ouabain 10000 -fold greater than that needed to inhibit fertilization. Although the spermatozoa were slowed in their motility by exposure to these high concentrations, after washing they fertilized oocytes with an efficiency equal to that of control spermatozoa. Similar findings were obtained by preincubation of oocytes. Ouabain therefore does not permanently injure the gametes, even at concentrations far in excess of those needed to block fertilization.

Although the best recognized action of ouabain is inhibition of $\mathrm{Na}^{+}-\mathrm{K}^{+}$ATPases (Garrahan \& Glynn, 1967; Jorgensen, 1982), and it does inhibit these enzymes in human (Peterson \& Freund, 1973), bull (McGrady, 1979), and boar (Uesugi \& Yamazoe, 1966) spermatozoa, the drug has other effects. Ouabain inhibits glycolysis in human spermatozoa at concentrations as low as $2 \times 10^{-6} \mathrm{M}$ (Peterson \& Freund, 1973), and lowers flagellar beat frequency and amplitude of bull spermatozoa at concentrations of ouabain greater than $10^{-6} \mathrm{M}$ (McGrady, 1979). However, $10^{-6} \mathrm{M}$-ouabain also reduces the membrane potential from -8.0 to $-5.6 \mathrm{mV}$ after $20 \mathrm{~min}$, an incubation time identical to the preincubation employed in our experiments (McGrady, 1979). Thus, while motility is changed slightly in bull spermatozoa at $10^{-6} \mathrm{M}$-ouabain, membrane potential is significantly altered.

That ouabain blocks fertilization by ion-pump inhibition in the present system is supported by limited studies with another $\mathrm{Na}^{+}-\mathrm{K}^{+}$ATPase inhibitor, digoxin. At a concentration of $100 \mu \mathrm{g} / \mathrm{ml}$ this substance blocked fertilization in $18 / 18$ zona-free oocytes exposed to capacitated, acrosomereacted spermatozoa. Because these drug concentrations are very high, the full set of controls performed with the ouabain studies were not extended to those with digoxin. The cumulative data support the idea that ion-pump inhibition with consequent alteration of membrane potential is the cause of the fertilization block.

If ouabain blocks fertilization by ATPase inhibition, it should be possible to demonstrate ATPase activity at the cell surface. Tests for ATPase activity at the surface of the unfertilized mammalian egg have not to our knowledge been conducted. Although ouabain-sensitive ATPases have previously been localized to the periacrosomal segment of the plasmalemma in cauda epididymal rabbit and guinea-pig spermatozoa (Gordon, 1977), similar attempts to localize ATPases in acrosome-reacted spermatozoa have not been made. The use of dbcGMP to produce homogeneous populations of functionally normal, acrosome-reacted spermatozoa has permitted us to undertake 
such studies. Preliminary results of these experiments demonstrate an ATPase at the surface of acrosome-reacted spermatozoa which is inhibited by ouabain (unpublished observations).

When inseminations were carried out after preincubation for $2 \mathrm{~h} 20 \mathrm{~min}$, spermatozoa treated with dbcGMP were less sensitive to ouabain than were controls. This suggests that the spermatozoon is one site of action of ouabain, though it again does not rule out effects on the oocyte. Even after $2 \mathrm{~h}$ of capacitation, spermatozoa treated with dbcGMP were more resistant to ouabain than were untreated spermatozoa. The reason for this difference is not known at this time.

In the presence of ouabain, spermatozoa bind avidly to the egg membrane but do not activate development. As shown in Fig. 2, extensive sperm binding takes place, but a second polar body is not released and a female pronucleus does not form. Ouabain therefore does not prevent sperm attachment; rather, it appears to block specific interactions which lead to oocyte activation after binding has occurred.

Acrosome-reaction induction as described here has a number of potential investigational and clinical applications. The ability to induce the acrosome reaction in nearly all spermatozoa of a given ejaculate should allow more detailed characterization of the acrosome-reacted spermatozoa, a cell type which is normally only a small component of a heterogeneous population. In the present study, induction of the acrosome reaction was exploited to demonstrate that mouse spermatozoa must have an intact acrosome at the time of zona contact if they are to penetrate oocytes. This possibly was suggested by previous time course studies of spermatozoa bound to the zona pellucida (Saling et al., 1979; Florman \& Storey, 1982; Storey et al., 1984). The use of dbcGMP has allowed us to establish a functionally significant relationship between retention of an intact acrosome and zona penetration.

Induction of the acrosome reaction might also confer fertility upon oligospermic males whose low sperm counts are associated with inadequate numbers of acrosome-reacted spermatozoa. Such applications would only be successful in species in which spermatozoa are able to penetrate the zona after first undergoing the acrosome reaction, or where holes placed in the zona allow direct access of spermatozoa to the oolemma (Gordon \& Talansky, 1986). One advantage of dbcGMP is that the prolonged incubation required by the lysolecithin-Ficoll protocol, which also induces the acrosome reaction (Fleming \& Yanagimachi, 1982), may not be necessary. We are now conducting time-course and concentration-dependency studies to characterize better the effects of dbcGMP, and determine its potential for improving the fertilizing capacity of ejaculated spermatozoa.

This work was supported by NIH grant no. HD18631 (J.W.G.). We thank Gregory S. Rudomen, CUNY, for excellent technical assistance.

\section{References}

Austin, C.R. (1951) Observations of the penetration of sperm into the mammalian egg. Aust. J. Sci. Res. 4, $581-589$.

Austin, C.R. (1952) The capacitation of the mammalian spermatozoa. Nature, Lond. 170, 326.

Austin, C.R. \& Bishop, M.W.H. (1958) Role of the rodent acrosome \& perforation in fertilization. Proc. R. Soc, B 149, 241-248.

Barg, P.E., Wahrman, M.Z., Talansky, B.E. \& Gordon, J.W. (1985) Capacitated, acrosome reacted but immotile sperm, when microinjected under the mouse zona pellucida, will not fertilize the oocyte. $J$. exp. Zool. 237, 365-374.

Chang, M.C. (1951) Fertilizing capacity of spermatozoa deposited into the fallopian tubes. Nature. Lond. 168, $697-698$.
Fleming, A.D. \& Yanagimachi, R. (1982) Fertile life of acrosome-reacted guinea pig spermatozoa. J. exp. Zool. 220, 109-115.

Florman, H.M. \& Storey, B.T. (1982) Mouse gamete interactions: The zona pellucida is the site of the acrosome reaction leading to fertilization in vitro. Devl Biol. 91, 121-130.

Garrahan, P.J. \& Glynn, I.M. (1967) Factors affecting the relative magnitudes of the sodium: potassium and sodium:sodium exchanges catalysed by the sodium pump. J. Physiol., Lond. 192, 189-216.

Gordon, J.W. \& Talansky, B.E. (1986) Assisted fertilization by zona drilling; a mouse model for correction of oligospermia. J. exp. Zool. 239, 347-354.

Gordon, M. (1977) Cytochemical analysis of the membranes of the mammalian sperm head. In Male 
Reproductive System, pp. 15-33. Eds R. Yates \& M. Gordon. Masson Publishing, Inc., New York.

Hoppe, P.C. \& Pitts, S. (1973) Fertilization in vitro and development of mouse ova. Biol. Reprod. 8, 420-426.

Jorgensen, P.L. (1982) Mechanism of the $\mathrm{Na}^{+}, \mathrm{K}^{+}$ pump. Protein structure conformation of the pure $\left(\mathrm{Na}^{+}, \mathrm{K}^{+}\right)$-ATPase. Biochim. biophys. Acta 694, $27-68$.

McGrady, A. (1979) The effect of ouabain on membrane potential and flagellar wave in ejaculated bull spermatozoa. J. Reprod. Fert. 56, 549-553.

Mrsny, R.J. \& Meizel, S. (1981) Potassium ion influx and $\mathrm{Na}^{+}, \mathrm{K}^{+}$-ATPase activity are required for the hamster sperm acrosome reaction. J. Cell Biol. 91, $77-82$.

Mrsny, R.J., Siiteri, J.E. \& Meizel, S. (1984) Hamster sperm $\mathrm{Na}^{+}, \mathrm{K}^{+}$-adenosine triphosphatase: increased activity during capacitation in vitro and its relationship to cyclic nucleotides. Biol. Reprod. 30, 573-584.

Nelson, L. \& McGrady, A.V. (1981) Effects of ouabain on spermatozoa function: a review. Archs Androl. 7, 169-176.

Peterson, R.N. \& Freund, M. (1973) Effects of $\left(\mathrm{H}^{+}\right)$, $\left(\mathrm{Na}^{+}\right),\left(\mathrm{K}^{+}\right)$and certain membrane-active drugs on glycolysis, motility, and ATP synthesis by human spermatozoa. Biol. Reprod. 8, 350-357.

Rogers, B.J. (1978) Mammalian sperm capacitation and fertilization in vitro: a critique of methodology. Gamete Res. 1, 165-223.

Saling, P.M., Sowinski, J. \& Storey, B.T. (1979) An ultrastructural study of epididymal mouse spermatozoa binding to zonae pellucidae in vitro: sequential relationship to the acrosome reaction. J. exp. Zool. 209, 229-238.

Santos-Sacchi, J. \& Gordon, M. (1980) Induction of the acrosome reaction in guinea pig spermatozoa by cGMP analogues. J. Cell Biol. 85, 798-803.

Storey, B.T., Lee, M.A., Muller, C., Ward, C.R. \& Wirtshafter, D.G. (1984) Binding of mouse spermatozoa to the zonae pellucidae of mouse eggs in cumulus: evidence that the acrosomes remain substantially intact. Biol. Reprod. 31, 1119-1128.

Thadani, V.M. (1982) Mice produced from eggs fertilized in vitro at a very low sperm:egg ratio. J. exp. Zool. 219, 277-283.

Uesugi, S. \& Yamazoe, S. (1966) Presence of sodium:potassium-stimulated ATPase in boar epididymal spermatozoa. Nature, Lond. 209, 403-404.

Wolf, D. (1977) Involvement of a trypsin-like activity in sperm penetration of zona-free mouse ova. $J$. exp. Zool. 199, 149-156.

Received 26 June 1986 